University of Nebraska - Lincoln

DigitalCommons@University of Nebraska - Lincoln

$10-25-2006$

\title{
Phase stability analysis using interval Newton method with NRTL model
}

Hatice Gecegormez

University of Mersin

Yasar Demirel

University of Nebraska-Lincoln, ydemirel2@unl.edu

Follow this and additional works at: https://digitalcommons.unl.edu/chemengfluidmech

Part of the Chemical Engineering Commons

Gecegormez, Hatice and Demirel, Yasar, "Phase stability analysis using interval Newton method with NRTL model" (2006). Papers in Fluid Mechanics. 2.

https://digitalcommons.unl.edu/chemengfluidmech/2

This Article is brought to you for free and open access by the Chemical and Biomolecular Engineering Research and Publications at DigitalCommons@University of Nebraska - Lincoln. It has been accepted for inclusion in Papers in Fluid Mechanics by an authorized administrator of DigitalCommons@University of Nebraska - Lincoln. 


\title{
Phase stability analysis using interval Newton method with NRTL model
}

\begin{abstract}
The Gibbs energy minimization using activity coefficient models and nonlinear equation solution techniques are commonly applied for phase stability problems. However, dependence on the initial estimates and multiple solutions for these highly nonlinear equations are common drawbacks for some of the conventional approaches. We have used interval Newton method with the local composition model of NRTL for the phase stability analysis of 10 binary systems and 2 ternary systems at various feed compositions to locate all the stationary points. Results indicate that the interval Newton method is reliable and efficient.
\end{abstract}

Keywords: Phase stability; Interval Newton method; NRTL model

Comment:- This paper was published in the Journal of "Fluid Phase Equilibria" Volume 232. and Issue1- 2 on 25 October 2005 ,pages 48-45. And all the copy rights (C) of this paper belong to Elsevier B.V. 


\section{Introduction}

Phase splitting due to thermodynamic instability of liquid mixtures plays important role in simulation and design problems of separation by distillation and extraction. In threephase distillation, for example, feed may become unstable and splits into two liquid phases at some stages in the column. To know the exact number of phases on a stage contributes considerably towards the mathematical stability of phase equilibria predictions [1] and [2]. Location of a feed point with respect to the binodal curve may be used to predict the number of exact phases in a ternary mixture of types I and II; only the feed points within the binodal curve will split into two liquid phases, and require threephase equilibrium calculations [3].

For some time, it has been a challenge to find a reliable stability analysis for fluid mixtures in any number of component and phases. Most commonly, stability analysis is based on the distance of tangent plane with respect to the Gibbs energy of mixing surface [4], [5], [6] and [7]. When the distance is negative then a mixture at specified temperature and pressure becomes unstable and splits into two liquid phases. Tangent plane distance function is the difference between the Gibbs free energy of a phase with composition $x$ and the tangent plane to the Gibbs free energy surface estimated from a candidate phase composition $z$. However, the tangent plane distance approach requires the solution of nonlinear equations obtained with the activity coefficient models or the equations of state. Such solutions may lead to multiple stationary points depending of the initial values or require the number of phases known [8], and sometimes may not be reliable [7].

Interval Newton method proposed about 10 years ago is another approach for the phase stability analysis [9], [10] and [11]. The method is a generalized bisection algorithm with some modifications so that it is relatively less sensitive to initial values, and should provide all the roots including global optimum [11]. The method has been tested with equation of states and activity coefficient models [9], [12] and [13], and for process design calculations, such as mixed flow reactor and a reaction kinetics model [14]. This study further tests the reliability of phase stability analysis for 10 binary mixtures and 2 
ternary mixtures using the interval Newton method and the activity coefficient model of NRTL, which might predict multiple roots in phase equilibrium calculations.

\section{Theoretical framework}

\subsection{Phase stability analysis}

The tangent plane distance $D$ for a mixture with $n$ number of components is expressed by:

$$
D(x)=g_{\mathrm{m}}(x)-g_{\mathrm{m}}(z)-\sum_{i=1}^{n}\left(\frac{\partial g_{\mathrm{m}}}{\partial x_{i}}\right)_{z}\left(x_{i}-z_{i}\right)
$$

Where $g_{\mathrm{m}}$ is the reduced Gibbs energy of mixing, and given by:

$$
\begin{aligned}
& g_{\mathrm{m}}(x)=\sum_{i=1}^{n} x_{i} \ln x_{i}+g^{\mathrm{E}}(x) \\
& g^{\mathrm{E}}=\frac{G^{\mathrm{E}}}{R T}
\end{aligned}
$$

where $g^{\mathrm{E}}$ is the reduced molar excess Gibbs energy. When the tangent plane distance $D$ for a composition $x$ is negative, a phase with feed mole fractions $z$ is unstable, and the molar Gibbs energy of mixing surface $g_{\mathrm{m}}=\Delta G_{\mathrm{mix}} / R T$ falls below a plane tangent to the surface at $z$. The partial derivatives in Eq. (1) are calculated at $x=z$. The tangent plane distance analysis minimizes Eq. (1), and the solutions of the following system of nonlinear equations identify the stationary points [13]:

$$
\begin{aligned}
& {\left[\left(\frac{\partial g_{\mathrm{m}}}{\partial x_{i}}\right)-\left(\frac{\partial g_{\mathrm{m}}}{\partial x_{n}}\right)\right]-\left[\left(\frac{\partial g_{\mathrm{m}}}{\partial x_{i}}\right)-\left(\frac{\partial g_{\mathrm{m}}}{\partial x_{n}}\right)\right]_{z}=0,} \\
& i=1, \ldots, n-1 \\
& \sum_{i=1}^{n} x_{i}=1
\end{aligned}
$$


The interval Newton/generalized bisection method requires no initial guess, and finds all the stationary points of the tangent plane distance $D$ by solving Eqs. (4) and (5) with some easy modifications, which are explained in detail by Hua et al. [9] and [10] and Gau and Stadtherr [14].

\subsection{NRTL model}

For an $n$ component system, the NRTL equation for the reduced molar excess Gibbs energy is given by:

$$
g^{\mathrm{E}}(x)=\sum_{k=1}^{n} x_{k} \frac{\sum_{i=1}^{n} G_{i k} \tau_{i k} x_{i}}{\sum_{i=1}^{n} G_{i k} x_{i}}
$$

where $x_{k}$ is the mole fraction for species $k$, and $\tau_{i k}$ and $G_{i k}$ are the NRTL binary interaction parameters. Eq. (6) uses the mole fraction weighted averages of the interaction parameters to improve the efficiency of the interval method [10] and [14]. The parameter $G_{i k}$ is a function of $\tau_{i k}$ and the parameter $\alpha_{i k}=\alpha_{k i}$, and is given by $G_{i k}=\exp \left(-\alpha_{i k} \tau_{i k}\right)$.With the NRTL model, Eq. (4) becomes [13]

$$
\ln \left(\frac{x_{i}}{x_{n}}\right)-\ln \left(\frac{z_{i}}{z_{n}}\right)+b_{i}(x)-b_{i}(z)=0, \quad i=1, \ldots, n-1
$$

where

$$
\begin{aligned}
b_{i}(x)= & \frac{\sum_{i=1}^{n-1} G_{i k} \tau_{i k} x_{i}}{\sum_{i=1}^{n-1} G_{i k} x_{i}}+\sum_{k=1}^{n} x_{k} \\
& \times\left[\frac{G_{i k} \tau_{i k}-G_{n k} \tau_{n k}}{\sum_{i=1}^{n} G_{i k} x_{i}}+\frac{\sum_{i=1}^{n} G_{i k} \tau_{i k} x_{i}\left(G_{n k}-G_{i k}\right)}{\left(\sum_{i=1}^{n} G_{i k} x_{i}\right)^{2}}\right]
\end{aligned}
$$

Eqs. (7) and (5) represent a set of $n \times n$ equation system whose solutions are the roots of $D$. 


\subsection{Interval Newton method}

The interval method is a general-purpose computational method to solve nonlinear equations to find all the solutions lying within the variable bounds [12]. It uses interval vectors and matrices starting with a specified initial box of intervals, and search all the roots by solving the linear interval equation system for a new interval $N^{(k)}$ :

$F^{\prime}\left(X^{(k)}\right)\left(N^{(k)}-x^{(k)}\right)=-f\left(x^{(k)}\right)$

Where $k$ is the iteration number, $F^{\prime}\left(X^{(k)}\right)$ is an interval extension of the real Jacobian of $f(x)$ over the current interval $\left(X^{(k)}\right)$ and $\left(x^{(k)}\right)$ is a midpoint of that interval. Tightness of the interval $\left(N^{(k)}\right)$ containing the solution set mostly depends on how to solve $\left(N^{(k)}\right)$. Usually, iterative methods with Gauss elimination is performed using preconditioned Eq. (9) for a tighter intervals [13] and [14]. Details of preconditioning of Eq. (9) and enhancing interval calculations are given by Schnepper and Stadtherr [12], Tessier et al. [13] and Gau and Stadtherr [14]. A reasonable initial box should be wide enough so that the interval Newton method provides all the solutions of local minima and maxima, saddle points and global minimum.

\section{Results and discussion}

We used 10 binary and 2 ternary systems for the stability analysis with the reduced Gibbs energy $g^{\mathrm{E}}$ obtained from the NRTL model. The local composition model of NRTL is known to have multiple roots in phase equilibrium calculations [2]. The systems include mostly the polar-polar mixtures. In order to test the method, we have obtained all the stationary points and the values of tangent plane distance $D$ for those roots from a MATLAB program. We verified the results of our MATLAB program with one of the binary systems used by Stadtherr and Schnepper [15] and a ternary system used by Tessier et al. [13]. The results of point approximations [13] presented in Table 1, Table 2, Table 3, Table 4, Table 5, Table 6, Table 7, Table 8, Table 9, Table 10, Table 11 and Table 12 represent the verified enclosures which contain a unique root $x$. The values of the tangent plane distance $D$ at each root have been obtained after satisfying that the deviations between the successive roots are as low as $1.00 \times 10^{-10}$. Table 1 , Table 2 , 
$\underline{\text { Table } 3}$, Table 4, Table 5, Table 6, Table 7, Table 8, Table 9 and Table 10 display the number of root inclusion tests and the solution times in seconds on a PC Genuine Intel computer, which is rather slow. The systems and the input data for the NRTL model are described in the following problems:

\section{Problem 1}

The binary parameters of polar-polar mixture $n$-pentanol(1)-2,2-dimethylbutane(2) are $\tau_{12}=1.0114044, \tau_{21}=2.9413053, G_{12}=0.5527024$ and $G_{21}=0.1782906$ [16]. Table 1 shows the values of $D$, which are negative at the stationary points $z_{1}=0.05$ and 0.10 , and the phases at these feed points are unstable. At the feed compositions $z_{1}=0.15$ and 0.20 , the phases are stable as $D>0$.

\section{Problem 2}

The binary parameters of polar-polar mixture $n$-pentanol(1)-2-methylpentane(2) are $\tau_{12}=0.9598699, \tau_{21}=2.9389421, G_{12}=0.5746116$ and $G_{21}=0.1833367$ [16]. As the values of $D$ in Table 2 show, the feed composition of $z_{1}=0.05,0.20$ and 0.25 are stable, while the feeds at $z_{1}=0.10,0.11$ and 0.12 are unstable.

\section{Problem 3}

The binary parameters of polar-apolar mixture ethanol(1)-cyclohexane(2) are $\tau_{12}=1.7252352, \tau_{21}=3.1963108, G_{12}=0.4541860$ and $G_{21}=0.2317199$ [16]. The values of $D$ in Table 3 show that the feeds at $z_{1}=0.10,0.15,0.20$ and 0.25 are unstable, while the feed compositions of $z_{1}=0.45,0.50,0.60$ and 0.65 are stable.

\section{Problem 4}

Water (1)-butyl glycol (2) mixture is a polar-polar mixture with the binary parameters of $\tau_{12}=1.2005955, \tau_{21}=1.4859846, G_{12}=0.8644289$ and $G_{21}=0.8350056$ [16]. The values of $D$ in Table 4 show that the feed points at $z_{1}=0.05$ and 0.10 are stable, while the feed points at $z_{1}=0.30,0.35,0.40,0.45,0.50,0.60$ and 0.65 are unstable. 


\section{Problem 5}

The binary parameters of polar-polar mixture water(1)-citric acid(2) are $\tau_{12}=0.9889317$, $\tau_{21}=13.7521382, G_{12}=0.6887706$ and $\mathrm{G}_{21}=5.6008823 \times 10^{-3}$ [17]. As seen in Table 5, the values of $D$ at the stationary points are positive and the feeds are stable.

\section{Problem 6}

The binary parameters of polar-polar mixture citric acid(1)-1-butanol(2) are $\tau_{12}=2.5479457 \times 10^{-2}, \tau_{21}=11.2949857, G_{12}=0.9948420$ and $G_{21}=0.1010207$ [17]. As Table 6 shows, the values of $D$ at the feed points $z_{1}=0.05,0.10,0.15,0.20$ and 0.25 are negative leading to phase instability. At the feed compositions $z_{1}=0.65$ and 0.75 , the system is stable with positive values of $D$.

\section{Problem 7}

The binary parameters of polar-polar mixture citric acid(1)-2-butanol(2) are $\tau_{12}=-1.3581754, \tau_{21}=7.4341774, G_{12}=1.3199010$ and $G_{21}=0.2188763$ [17]. As Table $\underline{7}$ shows, the feed points of $z_{1}=0.05,0.10,0.15,0.20$ and 0.25 are unstable. At the feed compositions $z_{1}=0.50,0.60,0.65$ and 0.75 the system is stable with positive values of $D$.

\section{Problem 8}

Water(1)-1,4-dicyanobutane(2) mixture is a polar-polar system, with the binary parameters of $\tau_{12}=4.6707725, \tau_{21}=0.6918617, G_{12}=0.2462934$ and $G_{21}=0.8125657$ [18]. The interval Newton method is successfully obtained 38 stationary points, which are shown in Table 8. At the feed compositions $z_{1}=0.05,0.10,0.15,0.20$ and 0.25 the system is stable with positive values of $D$. At the feed compositions $z_{1}=0.60,0.65$ and 0.75 the system is unstable with negative values of $D$.

\section{Problem 9}

The binary parameters of polar-polar mixture water(1)-butanenitrile(2) are $\tau_{12}=4.9011241, \tau_{21}=1.8395856, G_{12}=0.2298480$ and $G_{21}=0.5758687$ [18]. Table 9 shows that the feed point at $z_{1}=0.05$ is stable, while the feed points at $z_{1}=0.40,0.45$, 0.50 and 0.75 are unstable. 


\section{Problem 10}

The binary parameters of polar-polar mixture water(1)-benzonitrile(2) are $\tau_{12}=6.7197320, \tau_{21}=2.7710776, G_{12}=0.1331979$ and $G_{21}=0.4354727$ [18]. Table 10 shows that most of the feed points are unstable.

\section{Problem 11}

The ternary parameters for acetonitrile(1)-benzene(2)-n-heptane(3) mixture are $\tau_{12}=0.5661821, \tau_{21}=0.4472257, \tau_{23}=1.4918912, \tau_{32}=-0.5982783, \tau_{13}=2.3187177$, $\tau_{31}=0.6964173, G_{12}=0.9175650, G_{21}=0.9343016, G_{23}=0.6290071, G_{32}=1.2043230$, $G_{13}=0.7589032$ and $G_{31}=0.9204803$ [16]. Table 11 shows that the feed compositions are unstable.

\section{Problem 12}

The ternary parameters for water(1)-citric acid(2)-2-butanol(3) mixture are $\tau_{13}=2.9732685, \tau_{31}=0.5249036, G_{13}=0.2472300$ and $G_{31}=0.7813714$ [17]. The values of $\tau_{12}, \tau_{21}, G_{12}$ and $G_{21}$ are the same with those given in Problem 5, while the values of $\tau_{23}$, $\tau_{32}, G_{23}$ and $G_{32}$ are given in Problem 7. Table 12 shows the values of $D$ for stable and unstable feed compositions. 
Table 1.

Stationary points for $n$-pentanol(1)-2,2-dimethylbutane(2) system of Problem 1 at various feed points at $25^{\circ} \mathrm{C}$ and $1 \mathrm{~atm}$

\begin{tabular}{|c|c|c|c|c|}
\hline Feed $\left(z_{1}, z_{2}\right)$ & Stationary points $\left(x_{1}, x_{2}\right)$ & $D$ & Root inclusion tests & Solution time (s) \\
\hline \multirow[t]{2}{*}{$(0.05,0.95)$} & $(0.0500,0.9500)$ & 0.0000 & 399 & 38 \\
\hline & $(0.1294,0.8706)$ & $-1.8495 \times 10^{-3}$ & & \\
\hline \multirow[t]{3}{*}{$(0.10,0.90)$} & $\left(6.9187 \times 10^{-2}, 0.9308\right)$ & $-4.7473 \times 10^{-5}$ & 217 & 26 \\
\hline & $(0.1000,0.9000)$ & 0.0000 & & \\
\hline & $(0.1500,0.8500)$ & $-9.8214 \times 10^{-5}$ & & \\
\hline \multirow[t]{3}{*}{$(0.15,0.85)$} & $\left(6.9168 \times 10^{-2}, 0.9308\right)$ & $5.0013 \times 10^{-5}$ & 217 & 26 \\
\hline & $(0.1000,0.9000)$ & $9.7765 \times 10^{-5}$ & & \\
\hline & $(0.1500,0.8500)$ & 0.0000 & & \\
\hline \multirow[t]{2}{*}{$(0.20,0.80)$} & $\left(6.5086 \times 10^{-2}, 0.9349\right)$ & $2.9150 \times 10^{-3}$ & 209 & 25 \\
\hline & $(0.2000,0.8000)$ & 0.0000 & & \\
\hline
\end{tabular}




\section{Table 2.}

Stationary points for $n$-pentanol(1)-2-methylpentane(2) system of Problem 2 at various feed points at $25^{\circ} \mathrm{C}$ and $1 \mathrm{~atm}$

\begin{tabular}{|c|c|c|c|c|}
\hline Feed $\left(z_{1}, z_{2}\right)$ & Stationary points $\left(x_{1}, x_{2}\right)$ & $D$ & Root inclusion tests & Solution time (s) \\
\hline \multirow[t]{2}{*}{$(0.05,0.95)$} & $(0.0500,0.9500)$ & 0.0000 & 394 & 38 \\
\hline & $(0.1365,0.8635)$ & $2.1266 \times 10^{-3}$ & & \\
\hline \multirow[t]{3}{*}{$(0.10,0.90)$} & $\left(6.8845 \times 10^{-2}, 0.9311\right)$ & $-5.7673 \times 10^{-5}$ & 212 & 26 \\
\hline & $(0.1000,0.9000)$ & 0.0000 & & \\
\hline & $(0.1657,0.8343)$ & $-2.2122 \times 10^{-4}$ & & \\
\hline \multirow[t]{3}{*}{$(0.11,0.89)$} & $\left(6.5423 \times 10^{-2}, 0.9346\right)$ & $-1.4238 \times 10^{-4}$ & 222 & 26 \\
\hline & $(0.1100,0.8900)$ & 0.0000 & & \\
\hline & $(0.1582,0.8418)$ & $-9.3395 \times 10^{-5}$ & & \\
\hline \multirow[t]{3}{*}{$(0.12,0.88)$} & $\left(6.3345 \times 10^{-2}, 0.9367\right)$ & $-2.3299 \times 10^{-4}$ & 238 & 27 \\
\hline & $(0.1200,0.8800)$ & 0.0000 & & \\
\hline & $(0.1495,0.8505)$ & $-2.2301 \times 10^{-5}$ & & \\
\hline \multirow[t]{2}{*}{$(0.20,0.80)$} & $\left(7.0986 \times 10^{-2}, 0.9290\right)$ & $2.0998 \times 10^{-3}$ & 222 & 26 \\
\hline & $(0.2000,0.8000)$ & 0.0000 & & \\
\hline \multirow[t]{2}{*}{$(0.25,0.75)$} & $\left(5.5296 \times 10^{-2}, 0.9447\right)$ & $9.3157 \times 10^{-3}$ & 218 & 26 \\
\hline & $(0.2500,0.7500)$ & 0.0000 & & \\
\hline
\end{tabular}


Table 3.

Stationary points for ethanol(1)-cyclohexane(2) system of $\underline{\text { Problem } 3}$ at various feed points at $5{ }^{\circ} \mathrm{C}$ and $1 \mathrm{~atm}$

\begin{tabular}{|c|c|c|c|c|}
\hline Feed $\left(z_{1}, z_{2}\right)$ & Stationary points $\left(x_{1}, x_{2}\right)$ & $D$ & Root inclusion tests & Solution time (s) \\
\hline \multirow[t]{3}{*}{$(0.05,0.95)$} & $(0.0500,0.9500)$ & 0.0000 & 202 & 25 \\
\hline & $\left(9.4213 \times 10^{-2}, 0.9058\right)$ & $6.2615 \times 10^{-4}$ & & \\
\hline & $(0.5979,0.4021)$ & $-5.1329 \times 10^{-2}$ & & \\
\hline \multirow[t]{3}{*}{$(0.10,0.90)$} & $\left(4.7514 \times 10^{-2}, 0.9525\right)$ & $-1.0107 \times 10^{-3}$ & 187 & 23 \\
\hline & $(0.1000,0.9000)$ & 0.0000 & & \\
\hline & $(0.5878,0.4122)$ & $-4.8019 \times 10^{-2}$ & & \\
\hline \multirow[t]{4}{*}{$(0.15,0.85)$} & $\left(3.5205 \times 10^{-2}, 0.9648\right)$ & $-7.0829 \times 10^{-3}$ & 173 & 22 \\
\hline & $(0.1500,0.8500)$ & 0.0000 & & \\
\hline & $(0.4879,0.5121)$ & $-1.8081 \times 10^{-2}$ & & \\
\hline & $\left(0.9815,1.8550 \times 10^{-2}\right)$ & $-2.2408 \times 10^{-3}$ & & \\
\hline \multirow[t]{3}{*}{$(0.20,0.80)$} & $\left(3.0362 \times 10^{-2}, 0.9696\right)$ & $-1.4273 \times 10^{-2}$ & 170 & 22 \\
\hline & $(0.2000,0.8000)$ & 0.0000 & & \\
\hline & $(0.4000,0.6000)$ & $-4.0523 \times 10^{-3}$ & & \\
\hline \multirow[t]{3}{*}{$(0.25,0.75)$} & $\left(2.8470 \times 10^{-2}, 0.9715\right)$ & $-1.9142 \times 10^{-2}$ & 191 & 24 \\
\hline & $(0.2500,0.7500)$ & 0.0000 & & \\
\hline & $(0.3314,0.6686)$ & $-2.8350 \times 10^{-4}$ & & \\
\hline \multirow[t]{2}{*}{$(0.35,0.60)$} & $\left(2.8832 \times 10^{-2}, 0.9712\right)$ & $-1.7255 \times 10^{-2}$ & 197 & 24 \\
\hline & $(0.2351,0.7649)$ & $7.9166 \times 10^{-4}$ & & \\
\hline
\end{tabular}




\begin{tabular}{|c|c|c|c|c|}
\hline Feed $\left(z_{1}, z_{2}\right)$ & Stationary points $\left(x_{1}, x_{2}\right)$ & $D$ & Root inclusion tests & Solution time (s) \\
\hline & $(0.3500,0.6500)$ & 0.0000 & & \\
\hline \multirow[t]{3}{*}{$(0.40,0.55)$} & $\left(3.0363 \times 10^{-2}, 0.9696\right)$ & $-1.0218 \times 10^{-2}$ & 160 & 21 \\
\hline & $(0.2000,0.8000)$ & $4.0543 \times 10^{-3}$ & & \\
\hline & $(0.4000,0.6000)$ & 0.0000 & & \\
\hline \multirow[t]{4}{*}{$(0.45,0.60)$} & $\left(3.2732 \times 10^{-2}, 0.9673\right)$ & $5.4846 \times 10^{-4}$ & 179 & 22 \\
\hline & $(0.1702,0.8298)$ & $1.0652 \times 10^{-2}$ & & \\
\hline & $(0.4500,0.5500)$ & 0.0000 & & \\
\hline & $\left(0.9875,1.2543 \times 10^{-2}\right)$ & $2.8205 \times 10^{-2}$ & & \\
\hline \multirow[t]{4}{*}{$(0.50,0.50)$} & $\left(3.6146 \times 10^{-2}, 0.9638\right)$ & $1.4701 \times 10^{-2}$ & 174 & 22 \\
\hline & $(0.1439,0.8561)$ & $2.0885 \times 10^{-2}$ & & \\
\hline & $(0.5000,0.5000)$ & 0.0000 & & \\
\hline & $\left(0.9794,2.0600 \times 10^{-2}\right)$ & $1.1910 \times 10^{-2}$ & & \\
\hline \multirow[t]{3}{*}{$(0.60,0.40)$} & $\left(5.0600 \times 10^{-2}, 0.9495\right)$ & $5.2247 \times 10^{-2}$ & 196 & 24 \\
\hline & $\left(9.2933 \times 10^{-2}, 0.9071\right)$ & $5.2801 \times 10^{-2}$ & & \\
\hline & $(0.6000,0.4000)$ & 0.0000 & & \\
\hline \multirow[t]{2}{*}{$(0.65,0.35)$} & $\left(6.4067 \times 10^{-2}, 0.9359\right)$ & $7.5606 \times 10^{-2}$ & 285 & 32 \\
\hline & $(0.6500,0.3500)$ & 0.0000 & & \\
\hline \multirow[t]{3}{*}{$(0.75,0.25)$} & $\left(4.9309 \times 10^{-2}, 0.9507\right)$ & 0.1409 & 204 & 25 \\
\hline & $(0.7500,0.2500)$ & 0.0000 & & \\
\hline & $\left(0.9339,6.6045 \times 10^{-2}\right)$ & $-5.6707 \times 10^{-2}$ & & \\
\hline
\end{tabular}


Table 4.

Stationary points for water(1)-butyl glycol(2) system of Problem 4 at various feed points at $5{ }^{\circ} \mathrm{C}$ and 1 atm

\begin{tabular}{|c|c|c|c|c|}
\hline Feed $\left(z_{1}, z_{2}\right)$ & Stationary points $\left(x_{1}, x_{2}\right)$ & $D$ & Root inclusion tests & Solution time (s) \\
\hline \multirow[t]{2}{*}{$(0.05,0.95)$} & $(0.0500,0.9500)$ & 0.0000 & 265 & 30 \\
\hline & $(0.8403,0.1597)$ & 0.4708 & & \\
\hline \multirow[t]{2}{*}{$(0.10,0.90)$} & $(0.1000,0.9000)$ & 0.0000 & 404 & 39 \\
\hline & $(0.7234,0.2766)$ & 0.1255 & & \\
\hline \multirow[t]{3}{*}{$(0.15,0.85)$} & $(0.1500,0.8500)$ & 0.0000 & 178 & 22 \\
\hline & $(0.4768,0.5232)$ & $2.6938 \times 10^{-2}$ & & \\
\hline & $(0.8439,0.1561)$ & $-7.7671 \times 10^{-3}$ & & \\
\hline \multirow[t]{3}{*}{$(0.20,0.80)$} & $(0.2000,0.8000)$ & 0.0000 & 183 & 23 \\
\hline & $(0.3656,0.6344)$ & $3.9322 \times 10^{-3}$ & & \\
\hline & $(0.8709,0.1291)$ & $-7.0380 \times 10^{-2}$ & & \\
\hline \multirow[t]{3}{*}{$(0.25,0.75)$} & $(0.2500,0.7500)$ & 0.0000 & 213 & 26 \\
\hline & $(0.2956,0.7044)$ & $8.4999 \times 10^{-5}$ & & \\
\hline & $(0.8786,0.1214)$ & $-9.2025 \times 10^{-2}$ & & \\
\hline \multirow[t]{3}{*}{$(0.30,0.70)$} & $(0.2463,0.7537)$ & $-1.3869 \times 10^{-4}$ & 199 & 24 \\
\hline & $(0.3000,0.7000)$ & 0.0000 & & \\
\hline & $(0.8783,0.1217)$ & $-9.1484 \times 10^{-2}$ & & \\
\hline$(0.35,0.65)$ & $(0.2096,0.7904)$ & $-2.4208 \times 10^{-3}$ & 186 & 23 \\
\hline
\end{tabular}




\begin{tabular}{|c|c|c|c|c|}
\hline Feed $\left(z_{1}, z_{2}\right)$ & Stationary points $\left(x_{1}, x_{2}\right)$ & $\boldsymbol{D}$ & Root inclusion tests & Solution time (s) \\
\hline & $(0.3500,0.6500)$ & 0.0000 & & \\
\hline & $(0.8733,0.1267)$ & $-7.9379 \times 10^{-2}$ & & \\
\hline \multirow[t]{3}{*}{$(0.40,0.60)$} & $(0.1815,0.8185)$ & $-8.7794 \times 10^{-3}$ & 182 & 23 \\
\hline & $(0.4000,0.6000)$ & 0.0000 & & \\
\hline & $(0.8644,0.1356)$ & $-6.2151 \times 10^{-2}$ & & \\
\hline \multirow[t]{3}{*}{$(0.45,0.55)$} & $(0.1597,0.8403)$ & $-1.9546 \times 10^{-2}$ & 190 & 24 \\
\hline & $(0.4500,0.5500)$ & 0.0000 & & \\
\hline & $(0.8520,0.1480)$ & $-4.3912 \times 10^{-2}$ & & \\
\hline \multirow[t]{3}{*}{$(0.50,0.50)$} & $(0.1426,0.8574)$ & $-3.4091 \times 10^{-2}$ & 173 & 22 \\
\hline & $(0.5000,0.5000)$ & 0.0000 & & \\
\hline & $(0.8358,0.1642)$ & $-2.7355 \times 10^{-2}$ & & \\
\hline \multirow[t]{3}{*}{$(0.60,0.40)$} & $(0.1198,0.8802)$ & $-6.8384 \times 10^{-2}$ & 184 & 23 \\
\hline & $(0.6000,0.4000)$ & 0.0000 & & \\
\hline & $(0.7895,0.2105)$ & $-5.3761 \times 10^{-3}$ & & \\
\hline \multirow[t]{3}{*}{$(0.65,0.35)$} & $(0.1134,0.8866)$ & $-8.3043 \times 10^{-2}$ & 192 & 24 \\
\hline & $(0.6500,0.3500)$ & 0.0000 & & \\
\hline & $(0.7568,0.2432)$ & $-9.8759 \times 10^{-4}$ & & \\
\hline \multirow[t]{3}{*}{$(0.75,0.25)$} & $(0.1126,0.8874)$ & $-8.4479 \times 10^{-2}$ & 192 & 24 \\
\hline & $(0.6590,0.3410)$ & $6.1269 \times 10^{-4}$ & & \\
\hline & $(0.7500,0.2500)$ & 0.0000 & & \\
\hline
\end{tabular}


Table 5.

Stationary points for water(1)-citric acid(2) system of Problem 5 at various feed points at $25^{\circ} \mathrm{C}$ and $1 \mathrm{~atm}$

\begin{tabular}{|c|c|c|c|c|}
\hline Feed $\left(z_{1}, z_{2}\right)$ & Stationary points $\left(x_{1}, x_{2}\right)$ & $D$ & Root inclusion tests & Solution time (s) \\
\hline \multirow[t]{2}{*}{$(0.05,0.95)$} & $\left(1.0335 \times 10^{-2}, 0.9897\right)$ & $9.4569 \times 10^{-3}$ & 333 & 35 \\
\hline & $(0.0500,0.9500)$ & 0.0000 & & \\
\hline \multirow[t]{2}{*}{$(0.10,0.90)$} & $\left(7.1546 \times 10^{-3}, 0.9929\right)$ & $4.7783 \times 10^{-2}$ & 275 & 31 \\
\hline & $(0.1000,0.9000)$ & 0.0000 & & \\
\hline \multirow[t]{2}{*}{$(0.15,0.85)$} & $\left(5.9859 \times 10^{-3}, 0.9940\right)$ & $9.3235 \times 10^{-2}$ & 218 & 26 \\
\hline & $(0.1500,0.8500)$ & 0.0000 & & \\
\hline \multirow[t]{2}{*}{$(0.20,0.80)$} & $\left(5.3287 \times 10^{-3}, 0.9947\right)$ & 0.1412 & 180 & 22 \\
\hline & $(0.2000,0.8000)$ & 0.0000 & & \\
\hline \multirow[t]{2}{*}{$(0.25,0.75)$} & $\left(4.8886 \times 10^{-3}, 0.9951\right)$ & 0.1906 & 175 & 22 \\
\hline & $(0.2500,0.7500)$ & 0.0000 & & \\
\hline \multirow[t]{2}{*}{$(0.30,0.70)$} & $\left(4.5635 \times 10^{-3}, 0.9954\right)$ & 0.2413 & 172 & 22 \\
\hline & $(0.3000,0.7000)$ & 0.0000 & & \\
\hline \multirow[t]{3}{*}{$(0.35,0.65)$} & $\left(3.6100 \times 10^{-7}, 0.9999\right)$ & 0.2829 & 163 & 21 \\
\hline & $\left(4.3076 \times 10^{-3}, 0.9957\right)$ & 0.2933 & & \\
\hline & $(0.3500,0.6500)$ & 0.0000 & & \\
\hline \multirow[t]{2}{*}{$(0.40,0.60)$} & $\left(4.1700 \times 10^{-7}, 0.9999\right)$ & 0.3371 & 160 & 21 \\
\hline & $\left(4.0963 \times 10^{-3}, 0.9959\right)$ & 0.3469 & & \\
\hline
\end{tabular}




\begin{tabular}{|c|c|c|c|c|}
\hline Feed $\left(z_{1}, z_{2}\right)$ & Stationary points $\left(x_{1}, x_{2}\right)$ & $D$ & Root inclusion tests & Solution time (s) \\
\hline & $(0.4000,0.6000)$ & 0.0000 & & \\
\hline \multirow[t]{3}{*}{$(0.45,0.55)$} & $\left(4.7600 \times 10^{-7}, 0.9999\right)$ & 0.3934 & 161 & 21 \\
\hline & $\left(3.9150 \times 10^{-3}, 0.9961\right)$ & 0.4026 & & \\
\hline & $(0.4500,0.5500)$ & 0.0000 & & \\
\hline \multirow[t]{3}{*}{$(0.50,0.50)$} & $\left(5.4000 \times 10^{-7}, 0.9999\right)$ & 0.4525 & 153 & 21 \\
\hline & $\left(3.7541 \times 10^{-3}, 0.9963\right)$ & 0.4612 & & \\
\hline & $(0.5000,0.5000)$ & 0.0000 & & \\
\hline \multirow[t]{3}{*}{$(0.60,0.40)$} & $\left(6.8700 \times 10^{-7}, 0.9999\right)$ & 0.5845 & 150 & 21 \\
\hline & $\left(3.4665 \times 10^{-3}, 0.9965\right)$ & 0.5923 & & \\
\hline & $(0.6000,0.4000)$ & 0.0000 & & \\
\hline \multirow[t]{3}{*}{$(0.65,0.35)$} & $\left(7.7700 \times 10^{-7}, 0.9999\right)$ & 0.6618 & 159 & 21 \\
\hline & $\left(3.3288 \times 10^{-3}, 0.9967\right)$ & 0.6692 & & \\
\hline & $(0.6500,0.3500)$ & 0.0000 & & \\
\hline \multirow[t]{3}{*}{$(0.75,0.25)$} & $\left(1.0330 \times 10^{-6}, 0.9999\right)$ & 0.8605 & 159 & 21 \\
\hline & $\left(3.0361 \times 10^{-3}, 0.9970\right)$ & 0.8670 & & \\
\hline & $(0.7500,0.2500)$ & 0.0000 & & \\
\hline
\end{tabular}


Table 6.

Stationary points for citric acid(1)-1-butanol(2) system of $\underline{\text { Problem } 6}$ at various feed points at $25^{\circ} \mathrm{C}$ and $1 \mathrm{~atm}$

\begin{tabular}{|c|c|c|c|c|}
\hline Feed $\left(z_{1}, z_{2}\right)$ & Stationary points $\left(x_{1}, x_{2}\right)$ & $D$ & Root inclusion tests & Solution time (s) \\
\hline \multirow[t]{3}{*}{$(0.05,0.95)$} & $\left(7.6637 \times 10^{-5}, 0.9999\right)$ & $-8.2724 \times 10^{-2}$ & 237 & 27 \\
\hline & $(0.0500,0.9500)$ & 0.0000 & & \\
\hline & $\left(0.9516,4.8386 \times 10^{-2}\right)$ & -1.9587 & & \\
\hline \multirow[t]{3}{*}{$(0.10,0.90)$} & $\left(1.3061 \times 10^{-5}, 0.9999\right)$ & -0.2079 & 181 & 23 \\
\hline & $(0.1000,0.9000)$ & 0.0000 & & \\
\hline & $(0.7585,0.2415)$ & -0.5434 & & \\
\hline \multirow[t]{3}{*}{$(0.15,0.85)$} & $\left(6.1420 \times 10^{-6}, 0.9999\right)$ & -0.2995 & 168 & 22 \\
\hline & $(0.1500,0.8500)$ & 0.0000 & & \\
\hline & $(0.5596,0.4404)$ & -0.1325 & & \\
\hline \multirow[t]{3}{*}{$(0.20,0.80)$} & $\left(4.4010 \times 10^{-6}, 0.9999\right)$ & -0.3567 & 173 & 22 \\
\hline & $(0.2000,0.8000)$ & 0.0000 & & \\
\hline & $(0.4270,0.5730)$ & $-2.4001 \times 10^{-2}$ & & \\
\hline \multirow[t]{3}{*}{$(0.25,0.75)$} & $\left(3.8540 \times 10^{-6}, 0.9999\right)$ & -0.3859 & 190 & 24 \\
\hline & $(0.2500,0.7500)$ & 0.0000 & & \\
\hline & $(0.3422,0.6578)$ & $-1.6667 \times 10^{-3}$ & & \\
\hline \multirow[t]{3}{*}{$(0.30,0.70)$} & $\left(3.7530 \times 10^{-6}, 0.9999\right)$ & -0.3928 & 237 & 27 \\
\hline & $(0.2852,0.7148)$ & $7.0149 \times 10^{-6}$ & & \\
\hline & $(0.3000,0.7000)$ & 0.0000 & & \\
\hline
\end{tabular}




\begin{tabular}{|c|c|c|c|c|}
\hline Feed $\left(z_{1}, z_{2}\right)$ & Stationary points $\left(x_{1}, x_{2}\right)$ & $D$ & Root inclusion tests & Solution time (s) \\
\hline \multirow[t]{3}{*}{$(0.35,0.65)$} & $\left(3.8870 \times 10^{-6}, 0.9999\right)$ & -0.3813 & 188 & 23 \\
\hline & $(0.2444,0.7556)$ & $2.4989 \times 10^{-3}$ & & \\
\hline & $(0.3500,0.6500)$ & 0.0000 & & \\
\hline \multirow[t]{3}{*}{$(0.40,0.60)$} & $\left(4.1820 \times 10^{-6}, 0.9999\right)$ & -0.3537 & 175 & 22 \\
\hline & $(0.2137,0.7863)$ & $1.3448 \times 10^{-2}$ & & \\
\hline & $(0.40000,0.6000)$ & 0.0000 & & \\
\hline \multirow[t]{3}{*}{$(0.45,0.55)$} & $\left(4.6210 \times 10^{-6}, 0.9999\right)$ & -0.3113 & 172 & 22 \\
\hline & $(0.1895,0.8105)$ & $3.5874 \times 10^{-2}$ & & \\
\hline & $(0.4500,0.5500)$ & 0.0000 & & \\
\hline \multirow[t]{3}{*}{$(0.50,0.50)$} & $\left(5.2080 \times 10^{-6}, 0.9999\right)$ & -0.2544 & 170 & 22 \\
\hline & $(0.1697,0.8303)$ & $7.1332 \times 10^{-2}$ & & \\
\hline & $(0.5000,0.5000)$ & 0.0000 & & \\
\hline \multirow[t]{3}{*}{$(0.60,0.40)$} & $\left(6.9620 \times 10^{-6}, 0.9999\right)$ & $-9.4428 \times 10^{-2}$ & 173 & 22 \\
\hline & $(0.1384,0.8616)$ & 0.1871 & & \\
\hline & $(0.6000,0.4000)$ & 0.0000 & & \\
\hline \multirow[t]{3}{*}{$(0.65,0.35)$} & $\left(8.2660 \times 10^{-6}, 0.9999\right)$ & $1.2754 \times 10^{-2}$ & 174 & 22 \\
\hline & $(0.1253,0.8747)$ & 0.2717 & & \\
\hline & $(0.6500,0.3500)$ & 0.0000 & & \\
\hline$(0.75,0.25)$ & $\left(1.2528 \times 10^{-5}, 0.9999\right)$ & 0.3041 & 182 & 23 \\
\hline
\end{tabular}




\begin{tabular}{|l|l|l|l|l|}
\hline Feed $\left(\boldsymbol{z}_{\mathbf{1}}, \boldsymbol{z}_{\mathbf{2}}\right)$ & Stationary points $\left(\boldsymbol{x}_{\mathbf{1}}, \boldsymbol{x}_{\mathbf{2}}\right)$ & $\mathbf{D}$ & Root inclusion tests & Solution time (s) \\
\hline & $(0.1019,0.8981)$ & 0.5162 & & \\
\hline & $(0.7500,0.2500)$ & 0.0000 & & \\
\hline
\end{tabular}

Table 7.

Stationary points for citric acid(1)-2-butanol(2) system of Problem 7 at various feed points at $25{ }^{\circ} \mathrm{C}$ and $1 \mathrm{~atm}$

\begin{tabular}{|c|c|c|c|c|}
\hline Feed $\left(z_{1}, z_{2}\right)$ & Stationary points $\left(x_{1}, x_{2}\right)$ & $D$ & Root inclusion tests & Solution time (s) \\
\hline \multirow[t]{3}{*}{$(0.05,0.95)$} & $\left(7.0315 \times 10^{-3}, 0.9930\right)$ & $-9.6957 \times 10^{-3}$ & 201 & 25 \\
\hline & $(0.0500,0.9500)$ & 0.0000 & & \\
\hline & $(0.7096,0.2904)$ & -0.6614 & & \\
\hline \multirow[t]{3}{*}{$(0.10,0.90)$} & $\left(2.6555 \times 10^{-3}, 0.9973\right)$ & $-5.9344 \times 10^{-2}$ & 181 & 23 \\
\hline & $(0.1000,0.9000)$ & 0.0000 & & \\
\hline & $(0.5705,0.4295)$ & -0.2535 & & \\
\hline \multirow[t]{3}{*}{$(0.15,0.85)$} & $\left(1.5716 \times 10^{-3}, 0.9984\right)$ & -0.1147 & 168 & 22 \\
\hline & $(0.1500,0.8500)$ & 0.0000 & & \\
\hline & $(0.4558,0.5442)$ & $-7.3246 \times 10^{-2}$ & & \\
\hline \multirow[t]{3}{*}{$(0.20,0.80)$} & $\left(1.1991 \times 10^{-3}, 0.9988\right)$ & -0.1570 & 175 & 22 \\
\hline & $(0.2000,0.8000)$ & 0.0000 & & \\
\hline & $(0.3695,0.6305)$ & $-1.2904 \times 10^{-2}$ & & \\
\hline \multirow[t]{2}{*}{$(0.25,0.75)$} & $\left(1.0739 \times 10^{-3}, 0.9989\right)$ & -0.1795 & 188 & 23 \\
\hline & $(0.2500,0.7500)$ & 0.0000 & & \\
\hline
\end{tabular}




\begin{tabular}{|c|c|c|c|c|}
\hline Feed $\left(z_{1}, z_{2}\right)$ & Stationary points $\left(x_{1}, x_{2}\right)$ & $D$ & Root inclusion tests & Solution time (s) \\
\hline & $(0.3046,0.6956)$ & $-4.3191 \times 10^{-4}$ & & \\
\hline \multirow[t]{3}{*}{$(0.30,0.70)$} & $\left(1.0701 \times 10^{-3}, 0.9989\right)$ & -0.1800 & 197 & 24 \\
\hline & $(0.2539,0.7461)$ & $2.6409 \times 10^{-4}$ & & \\
\hline & $(0.3000,0.7000)$ & 0.0000 & & \\
\hline \multirow[t]{3}{*}{$(0.35,0.65)$} & $\left(1.1485 \times 10^{-3}, 0.9989\right)$ & -0.1585 & 175 & 22 \\
\hline & $(0.2136,0.7864)$ & $6.7593 \times 10^{-3}$ & & \\
\hline & $(0.3500,0.6500)$ & 0.0000 & & \\
\hline \multirow[t]{3}{*}{$(0.40,0.60)$} & $\left(1.3013 \times 10^{-3}, 0.9987\right)$ & -0.1151 & 168 & 22 \\
\hline & $(0.1806,0.8194)$ & $2.7671 \times 10^{-2}$ & & \\
\hline & $(0.4000,0.6000)$ & 0.0000 & & \\
\hline \multirow[t]{3}{*}{$(0.45,0.55)$} & $\left(1.5380 \times 10^{-3}, 0.9985\right)$ & $-5.0293 \times 10^{-2}$ & 167 & 22 \\
\hline & $(0.1529,0.8471)$ & $6.7348 \times 10^{-2}$ & & \\
\hline & $(0.4500,0.5500)$ & 0.0000 & & \\
\hline \multirow[t]{3}{*}{$(0.50,0.50)$} & $\left(1.8835 \times 10^{-3}, 0.9981\right)$ & $3.5767 \times 10^{-2}$ & 194 & 24 \\
\hline & $(0.1291,0.8709)$ & 0.1282 & & \\
\hline & $(0.5000,0.5000)$ & 0.0000 & & \\
\hline \multirow[t]{3}{*}{$(0.60,0.40)$} & $\left(3.1335 \times 10^{-3}, 0.9969\right)$ & 0.2739 & 185 & 23 \\
\hline & $\left(8.8965 \times 10^{-2}, 0.9110\right)$ & 0.3207 & & \\
\hline & $(0.6000,0.4000)$ & 0.0000 & & \\
\hline
\end{tabular}




\begin{tabular}{|l|l|l|l|l|}
\hline Feed $\left(\boldsymbol{z}_{\mathbf{1}}, \boldsymbol{z}_{\mathbf{2}}\right)$ & Stationary points $\left(\boldsymbol{x}_{\mathbf{1}}, \boldsymbol{x}_{\mathbf{2}}\right)$ & $\boldsymbol{D}$ & Root inclusion tests & Solution time (s) \\
\hline$(0.65,0.35)$ & $\left(4.3155 \times 10^{-3}, 0.9957\right)$ & 0.4291 & 178 & 22 \\
\hline & $\left(7.1117 \times 10^{-2}, 0.9289\right)$ & 0.4569 & & \\
\hline & $(0.6500,0.3500)$ & 0.0000 & & \\
\hline & & & \\
\hline$(0.75,0.25)$ & $\left(1.1696 \times 10^{-2}, 0.9883\right)$ & 0.8308 & 229 & \\
\hline & $\left(3.3412 \times 10^{-2}, 0.9666\right)$ & 0.8323 & & \\
\hline & $(0.7500,0.2500)$ & 0.0000 & & \\
\hline
\end{tabular}

\section{Table 8.}

Stationary points for water(1)-1,4-dicyanobutane(2) system of Problem 8 at various feed points at $25^{\circ} \mathrm{C}$ and $1 \mathrm{~atm}$

\begin{tabular}{|c|c|c|c|c|}
\hline Feed $\left(z_{1}, z_{2}\right)$ & Stationary points $\left(x_{1}, x_{2}\right)$ & $D$ & Root inclusion tests & Solution time (s) \\
\hline \multirow[t]{2}{*}{$(0.05,0.95)$} & $(0.0500,0.9500)$ & 0.0000 & 299 & 33 \\
\hline & $\left(0.9828,1.7225 \times 10^{-2}\right)$ & 1.2358 & & \\
\hline \multirow[t]{3}{*}{$(0.10,0.90)$} & $(0.1000,0.9000)$ & 0.0000 & 254 & 29 \\
\hline & $\left(0.9294,7.0586 \times 10^{-2}\right)$ & 0.6703 & & \\
\hline & $\left(0.9822,1.7844 \times 10^{-2}\right)$ & 0.6648 & & \\
\hline \multirow[t]{3}{*}{$(0.15,0.85)$} & $(0.1500,0.8500)$ & 0.0000 & 223 & 26 \\
\hline & $(0.8787,0.1213)$ & 0.3997 & & \\
\hline & $\left(0.9904,9.5702 \times 10^{-3}\right)$ & 0.3657 & & \\
\hline \multirow[t]{3}{*}{$(0.20,0.80)$} & $(0.2000,0.8000)$ & 0.0000 & 205 & 25 \\
\hline & $(0.8381,0.1619)$ & 0.2425 & & \\
\hline & $\left(0.9931,6.9371 \times 10^{-3}\right)$ & 0.1780 & & \\
\hline
\end{tabular}




\begin{tabular}{|c|c|c|c|c|}
\hline Feed $\left(z_{1}, z_{2}\right)$ & Stationary points $\left(x_{1}, x_{2}\right)$ & $D$ & Root inclusion tests & Solution time (s) \\
\hline \multirow[t]{3}{*}{$(0.25,0.75)$} & $(0.2500,0.7500)$ & 0.0000 & 195 & 24 \\
\hline & $(0.8005,0.1995)$ & 0.1441 & & \\
\hline & $\left(0.9944,5.6094 \times 10^{-3}\right)$ & $5.0924 \times 10^{-2}$ & & \\
\hline \multirow[t]{3}{*}{$(0.30,0.70)$} & $(0.3000,0.7000)$ & 0.0000 & 188 & 23 \\
\hline & $(0.7638,0.2362)$ & $8.1133 \times 10^{-2}$ & & \\
\hline & $\left(0.9952,4.8177 \times 10^{-3}\right)$ & $-3.8166 \times 10^{-2}$ & & \\
\hline \multirow[t]{3}{*}{$(0.35,0.65)$} & $(0.3500,0.6500)$ & 0.0000 & 183 & 23 \\
\hline & $(0.7267,0.2733)$ & $4.1516 \times 10^{-2}$ & & \\
\hline & $\left(0.9957,4.3056 \times 10^{-3}\right)$ & -0.1012 & & \\
\hline \multirow[t]{3}{*}{$(0.40,0.60)$} & $(0.4000,0.6000)$ & 0.0000 & 183 & 23 \\
\hline & $(0.6882,0.3118)$ & $1.7966 \times 10^{-2}$ & & \\
\hline & $\left(0.9960,3.9635 \times 10^{-3}\right)$ & -0.1449 & & \\
\hline \multirow[t]{3}{*}{$(0.45,0.55)$} & $(0.4500,0.5500)$ & 0.0000 & 205 & 25 \\
\hline & $(0.6475,0.3525)$ & $5.6431 \times 10^{-3}$ & & \\
\hline & $\left(0.9963,3.7390 \times 10^{-3}\right)$ & -1.7361 & & \\
\hline \multirow[t]{3}{*}{$(0.50,0.50)$} & $(0.5000,0.5000)$ & 0.0000 & 217 & 26 \\
\hline & $(0.6037,0.3963)$ & $8.0606 \times 10^{-4}$ & & \\
\hline & $\left(0.9964,3.6072 \times 10^{-3}\right)$ & -0.1899 & & \\
\hline$(0.60,0.40)$ & $(0.5041,0.4959)$ & $-6.3609 \times 10^{-4}$ & 219 & 26 \\
\hline
\end{tabular}




\begin{tabular}{|l|l|l|l|l|}
\hline Feed $\left(\boldsymbol{z}_{\mathbf{1}}, \boldsymbol{z}_{\mathbf{2}}\right)$ & Stationary points $\left(\boldsymbol{x}_{\mathbf{1}}, \boldsymbol{x}_{\mathbf{2}}\right)$ & $\boldsymbol{D}$ & Root inclusion tests & Solution time (s) \\
\hline & $(0.6000,0.4000)$ & 0.0000 & & \\
\hline & $\left(0.9964,3.6001 \times 10^{-3}\right)$ & -0.1914 & & \\
\hline$(0.65,0.35)$ & $(0.4470,0.5530)$ & $-6.1343 \times 10^{-3}$ & 205 & \\
\hline & $(0.6500,0.3500)$ & 0.0000 & & \\
\hline & $\left(0.9963,3.7496 \times 10^{-3}\right)$ & -0.1784 & & \\
\hline & $(0.3187,0.6813)$ & $-6.4009 \times 10^{-2}$ & 188 & \\
\hline$(0.75,0.25)$ & $(0.7500,0.2500)$ & 0.0000 & & \\
\hline & $\left(0.9954,4.6007 \times 10^{-3}\right)$ & -0.1284 & & \\
\hline
\end{tabular}

Table 9.

Stationary points for water(1)-butanenitrile(2) system of $\underline{\text { Problem } 9}$ at various feed points at $25{ }^{\circ} \mathrm{C}$ and $1 \mathrm{~atm}$

\begin{tabular}{|c|c|c|c|c|}
\hline Feed $\left(z_{1}, z_{2}\right)$ & Stationary points $\left(x_{1}, x_{2}\right)$ & $D$ & Root inclusion tests & Solution time (s) \\
\hline \multirow[t]{4}{*}{$(0.05,0.95)$} & $(0.0500,0.9500)$ & 0.0000 & 255 & 29 \\
\hline & $(0.2423,0.7577)$ & 0.2398 & & \\
\hline & $(0.8199,0.1801)$ & 0.4452 & & \\
\hline & $\left(0.9958,4.1846 \times 10^{-3}\right)$ & 0.3478 & & \\
\hline \multirow[t]{4}{*}{$(0.10,0.90)$} & $(0.1000,0.9000)$ & 0.0000 & 227 & 26 \\
\hline & $(0.3612,0.6388)$ & 0.1374 & & \\
\hline & $(0.6923,0.3077)$ & 0.1369 & & \\
\hline & $\left(0.9975,2.5029 \times 10^{-3}\right)$ & $-6.3535 \times 10^{-2}$ & & \\
\hline \multirow[t]{2}{*}{$(0.15,0.85)$} & $(0.1500,0.8500)$ & 0.0000 & 213 & 26 \\
\hline & $(0.5810,0.4190)$ & $4.2080 \times 10^{-2}$ & & \\
\hline
\end{tabular}




\begin{tabular}{|c|c|c|c|c|}
\hline Feed $\left(z_{1}, z_{2}\right)$ & Stationary points $\left(x_{1}, x_{2}\right)$ & $D$ & Root inclusion tests & Solution time (s) \\
\hline & $\left(0.9979,2.0467 \times 10^{-3}\right)$ & -0.2230 & & \\
\hline \multirow[t]{3}{*}{$(0.20,0.80)$} & $(0.2000,0.8000)$ & 0.0000 & 199 & 24 \\
\hline & $(0.4835,0.5165)$ & $1.0652 \times 10^{-2}$ & & \\
\hline & $\left(0.9981,1.8634 \times 10^{-3}\right)$ & -0.2942 & & \\
\hline \multirow[t]{3}{*}{$(0.25,0.75)$} & $(0.2500,0.7500)$ & 0.0000 & 207 & 25 \\
\hline & $(0.4034,0.5966)$ & $1.6082 \times 10^{-3}$ & & \\
\hline & $\left(0.9982,1.7843 \times 10^{-3}\right)$ & -0.3253 & & \\
\hline \multirow[t]{3}{*}{$(0.30,0.70)$} & $(0.3000,0.7000)$ & 0.0000 & 239 & 27 \\
\hline & $(0.3407,0.6593)$ & $2.9584 \times 10^{-5}$ & & \\
\hline & $\left(0.9982,1.7564 \times 10^{-3}\right)$ & -0.3359 & & \\
\hline \multirow[t]{3}{*}{$(0.40,0.60)$} & $(0.2524,0.7476)$ & $-1.4309 \times 10^{-3}$ & 212 & 26 \\
\hline & $(0.4000,0.6000)$ & 0.0000 & & \\
\hline & $\left(0.9982,1.7821 \times 10^{-3}\right)$ & -0.3276 & & \\
\hline \multirow[t]{3}{*}{$(0.45,0.55)$} & $(0.2195,0.7805)$ & $-5.5825 \times 10^{-3}$ & 209 & 25 \\
\hline & $(0.4500,0.5500)$ & 0.0000 & & \\
\hline & $\left(0.9982,1.8242 \times 10^{-3}\right)$ & -0.3153 & & \\
\hline \multirow[t]{3}{*}{$(0.50,0.50)$} & $(0.1909,0.8091)$ & $-1.4010 \times 10^{-2}$ & 204 & 25 \\
\hline & $(0.5000,0.5000)$ & 0.0000 & & \\
\hline & $\left(0.9981,1.8865 \times 10^{-3}\right)$ & -0.2991 & & \\
\hline
\end{tabular}




\begin{tabular}{|c|c|c|c|c|}
\hline Feed $\left(z_{1}, z_{2}\right)$ & Stationary points $\left(x_{1}, x_{2}\right)$ & $\boldsymbol{D}$ & Root inclusion tests & Solution time (s) \\
\hline \multirow[t]{4}{*}{$(0.60,0.40)$} & $(0.1411,0.8589)$ & $-5.2405 \times 10^{-2}$ & 209 & 25 \\
\hline & $(0.4099,0.5901)$ & $4.2647 \times 10^{-2}$ & & \\
\hline & $(0.6000,0.4000)$ & 0.0000 & & \\
\hline & $\left(0.9979,2.0994 \times 10^{-3}\right)$ & -0.2557 & & \\
\hline \multirow[t]{4}{*}{$(0.65,0.35)$} & $(0.1184,0.8816)$ & $-8.9759 \times 10^{-2}$ & 225 & 26 \\
\hline & $(0.3857,0.6143)$ & $2.5465 \times 10^{-2}$ & & \\
\hline & $(0.6500,0.3500)$ & 0.0000 & & \\
\hline & $\left(0.9977,2.2805 \times 10^{-3}\right)$ & -0.2278 & & \\
\hline \multirow[t]{4}{*}{$(0.75,0.25)$} & $\left(7.6194 \times 10^{-2}, 0.9238\right)$ & -0.2366 & 251 & 29 \\
\hline & $(0.3185,0.6815)$ & $-5.9660 \times 10^{-2}$ & & \\
\hline & $(0.7500,0.2500)$ & 0.0000 & & \\
\hline & $\left(0.9970,2.9876 \times 10^{-3}\right)$ & -0.1575 & & \\
\hline
\end{tabular}

\section{Table 10.}

Stationary points for water(1)-benzonitrile(2) system of Problem 10 at various feed points at $25^{\circ} \mathrm{C}$ and $1 \mathrm{~atm}$

\begin{tabular}{|c|c|c|c|c|}
\hline Feed $\left(z_{1}, z_{2}\right)$ & Stationary points $\left(x_{1}, x_{2}\right)$ & $D$ & Root inclusion tests & Solution time (s) \\
\hline \multirow[t]{3}{*}{$(0.05,0.95)$} & $(0.0500,0.9500)$ & 0.0000 & 266 & 30 \\
\hline & $(0.7513,0.2487)$ & 0.1205 & & \\
\hline & $\left(0.9997,3.2783 \times 10^{-4}\right)$ & $-9.2920 \times 10^{-2}$ & & \\
\hline \multirow[t]{3}{*}{$(0.10,0.90)$} & $(0.1000,0.9000)$ & 0.0000 & 264 & 30 \\
\hline & $(0.2383,0.7617)$ & $-3.5325 \times 10^{-3}$ & & \\
\hline & $\left(0.9997,2.5340 \times 10^{-4}\right)$ & -0.3258 & & \\
\hline
\end{tabular}




\begin{tabular}{|c|c|c|c|c|}
\hline Feed $\left(z_{1}, z_{2}\right)$ & Stationary points $\left(x_{1}, x_{2}\right)$ & $D$ & Root inclusion tests & Solution time (s) \\
\hline \multirow[t]{3}{*}{$(0.20,0.80)$} & $(0.1156,0.8844)$ & $-9.0701 \times 10^{-4}$ & 266 & 30 \\
\hline & $(0.2000,0.8000)$ & 0.0000 & & \\
\hline & $\left(0.9997,2.4742 \times 10^{-4}\right)$ & -0.3475 & & \\
\hline \multirow[t]{3}{*}{$(0.25,0.75)$} & $\left(9.6405 \times 10^{-2}, 0.9036\right)$ & $-4.6336 \times 10^{-3}$ & 242 & 28 \\
\hline & $(0.2500,0.7500)$ & 0.0000 & & \\
\hline & $\left(0.9997,2.5537 \times 10^{-4}\right)$ & -0.3236 & & \\
\hline \multirow[t]{3}{*}{$(0.30,0.70)$} & $\left(8.5011 \times 10^{-2}, 0.9150\right)$ & $-1.0332 \times 10^{-2}$ & 225 & 26 \\
\hline & $(0.3000,0.7000)$ & 0.0000 & & \\
\hline & $\left(0.9997,2.6362 \times 10^{-4}\right)$ & -0.3012 & & \\
\hline \multirow[t]{3}{*}{$(0.35,0.65)$} & $\left(7.7947 \times 10^{-2}, 0.9221\right)$ & $-1.6598 \times 10^{-2}$ & 212 & 26 \\
\hline & $(0.3500,0.6500)$ & 0.0000 & & \\
\hline & $\left(0.9997,2.7071 \times 10^{-4}\right)$ & -0.2837 & & \\
\hline \multirow[t]{3}{*}{$(0.40,0.60)$} & $\left(7.3539 \times 10^{-2}, 0.9265\right)$ & $-2.2359 \times 10^{-2}$ & 231 & 27 \\
\hline & $(0.4000,0.6000)$ & 0.0000 & & \\
\hline & $\left(0.9997,2.7614 \times 10^{-4}\right)$ & -0.2716 & & \\
\hline \multirow[t]{3}{*}{$(0.45,0.55)$} & $\left(7.0822 \times 10^{-2}, 0.9292\right)$ & $-2.7046 \times 10^{-2}$ & 221 & 26 \\
\hline & $(0.4500,0.5500)$ & 0.0000 & & \\
\hline & $\left(0.9997,2.7996 \times 10^{-4}\right)$ & -0.2639 & & \\
\hline$(0.50,0.50)$ & $\left(6.9111 \times 10^{-2}, 0.9309\right)$ & $-3.0688 \times 10^{-2}$ & 217 & 26 \\
\hline
\end{tabular}




\begin{tabular}{|c|c|c|c|c|}
\hline Feed $\left(z_{1}, z_{2}\right)$ & Stationary points $\left(x_{1}, x_{2}\right)$ & $D$ & Root inclusion tests & Solution time (s) \\
\hline & $(0.5000,0.5000)$ & 0.0000 & & \\
\hline & $\left(0.9997,2.8257 \times 10^{-4}\right)$ & -0.2592 & & \\
\hline \multirow[t]{3}{*}{$(0.60,0.40)$} & $\left(6.6090 \times 10^{-2}, 0.9339\right)$ & $-3.9061 \times 10^{-2}$ & 217 & 26 \\
\hline & $(0.6000,0.4000)$ & 0.0000 & & \\
\hline & $\left(0.9997,2.8762 \times 10^{-4}\right)$ & -0.2515 & & \\
\hline \multirow[t]{3}{*}{$(0.65,0.35)$} & $\left(6.3181 \times 10^{-2}, 0.9368\right)$ & $-4.9320 \times 10^{-2}$ & 220 & 26 \\
\hline & $(0.6500,0.3500)$ & 0.0000 & & \\
\hline & $\left(0.9997,2.9306 \times 10^{-4}\right)$ & -0.2447 & & \\
\hline \multirow[t]{3}{*}{$(0.75,0.25)$} & $\left(5.0253 \times 10^{-2}, 0.9497\right)$ & -0.1187 & 253 & 29 \\
\hline & $(0.7500,0.2500)$ & 0.0000 & & \\
\hline & $\left(0.9997,3.2696 \times 10^{-4}\right)$ & -0.2141 & & \\
\hline
\end{tabular}

\section{Table 11.}

Stationary points for acetonitrile(1)-benzene(2)- $n$-heptane(3) system of Problem 11 at various feed points at $45^{\circ} \mathrm{C}$ and $1 \mathrm{~atm}$

\begin{tabular}{|l|l|l|}
\hline Feed $\left(z_{1}, z_{2}, z_{3}\right)$ & Stationary points $\left(x_{1}, x_{2}, x_{3}\right)$ & $D$ \\
\hline$(0.40,0.05,0.55)$ & $\left(0.2215,4.8013 \times 10^{-2}, 0.7304\right)$ & $-5.1389 \times 10^{-3}$ \\
\hline & $(0.4000,0.0500,0.5500)$ & 0.0000 \\
\hline \multicolumn{2}{|l}{} \\
\hline$(0.45,0.05,0.50)$ & $\left(0.1919,4.7275 \times 10^{-2}, 0.7608\right)$ & $-1.5255 \times 10^{-2}$ \\
\hline & $(0.4500,0.0500,0.5000)$ & 0.0000 \\
\hline
\end{tabular}




\begin{tabular}{|c|c|c|}
\hline Feed $\left(z_{1}, z_{2}, z_{3}\right)$ & Stationary points $\left(x_{1}, x_{2}, x_{3}\right)$ & $D$ \\
\hline \multirow[t]{2}{*}{$(0.60,0.05,0.35)$} & $\left(0.1320,4.6723 \times 10^{-2}, 0.8213\right)$ & $-8.0816 \times 10^{-2}$ \\
\hline & $(0.6000,0.0500,0.3500)$ & 0.0000 \\
\hline \multirow[t]{2}{*}{$(0.70,0.05,0.25)$} & $\left(0.1118,4.9260 \times 10^{-2}, 0.8389\right)$ & -0.1334 \\
\hline & $(0.7000,0.0500,0.2500)$ & 0.0000 \\
\hline \multirow[t]{2}{*}{$(0.50,0.10,0.40)$} & $\left(0.1720,9.5257 \times 10^{-2}, 0.7327\right)$ & $-2.8631 \times 10^{-2}$ \\
\hline & $(0.5000,0.1000,0.4000)$ & 0.0000 \\
\hline \multirow[t]{2}{*}{$(0.55,0.10,0.35)$} & $\left(0.1536,9.5606 \times 10^{-2}, 0.7508\right)$ & $-4.7718 \times 10^{-2}$ \\
\hline & $(0.5500,0.1000,0.3500)$ & 0.0000 \\
\hline \multirow[t]{2}{*}{$(0.65,0.10,0.25)$} & $(0.1309,0.1006,0.7685)$ & $-8.6916 \times 10^{-2}$ \\
\hline & $(0.6500,0.1000,0.2500)$ & 0.0000 \\
\hline \multirow[t]{2}{*}{$(0.45,0.15,0.40)$} & $(0.2023,0.1460,0.6518)$ & $-1.1811 \times 10^{-2}$ \\
\hline & $(0.4500,0.1500,0.4000)$ & 0.0000 \\
\hline \multirow[t]{2}{*}{$(0.50,0.15,0.35)$} & $(0.1805,0.1465,0.6731)$ & $-2.3794 \times 10^{-2}$ \\
\hline & $(0.5000,0.1500,0.3500)$ & 0.0000 \\
\hline \multirow[t]{2}{*}{$(0.60,0.15,0.25)$} & $(0.1548,0.1538,0.6914)$ & $-5.0477 \times 10^{-2}$ \\
\hline & $(0.6000,0.1500,0.2500)$ & 0.0000 \\
\hline \multirow[t]{2}{*}{$(0.45,0.20,0.35)$} & $(0.2154,0.1989,0.5857)$ & $-8.6429 \times 10^{-3}$ \\
\hline & $(0.4500,0.2000,0.3500)$ & 0.0000 \\
\hline
\end{tabular}




\begin{tabular}{|l|l|l|}
\hline Feed $\left(\boldsymbol{z}_{1}, \boldsymbol{z}_{2}, \boldsymbol{z}_{3}\right)$ & Stationary points $\left(\boldsymbol{x}_{\mathbf{1}}, \boldsymbol{x}_{\mathbf{2}}, \boldsymbol{x}_{\mathbf{3}}\right)$ & $\mathrm{D}$ \\
\hline & \multicolumn{2}{|l|}{} \\
\hline$(0.55,0.20,0.25)$ & $(0.1863,0.2085,0.6051)$ & $-2.4032 \times 10^{-2}$ \\
\hline & $(0.5500,0.2000,0.2500)$ & 0.0000 \\
\hline
\end{tabular}

Table 12.

Stationary points for water(1)-citric acid(2)-2-butanol(3) system of Problem 12 at various feed points at $25^{\circ} \mathrm{C}$ and $1 \mathrm{~atm}$

\begin{tabular}{|c|c|c|}
\hline Feed $\left(z_{1}, z_{2}, z_{3}\right)$ & Stationary points $\left(x_{1}, x_{2}, x_{3}\right)$ & $D$ \\
\hline \multirow[t]{2}{*}{$(0.10,0.05,0.85)$} & $\left(6.9754 \times 10^{-2}, 1.0768 \times 10^{-2}, 0.9195\right)$ & $-4.4700 \times 10^{-3}$ \\
\hline & $(0.1000,0.0500,0.8500)$ & 0.0000 \\
\hline \multirow[t]{3}{*}{$(0.20,0.05,0.75)$} & $\left(0.1752,2.7558 \times 10^{-2}, 0.7972\right)$ & $-2.3699 \times 10^{-4}$ \\
\hline & $(0.2000,0.0500,0.7500)$ & 0.0000 \\
\hline & $(0.2530,0.1748,0.5722)$ & $-5.9447 \times 10^{-3}$ \\
\hline \multirow[t]{2}{*}{$(0.25,0.05,0.70)$} & $(0.2500,0.0500,0.7000)$ & 0.0000 \\
\hline & $\left(0.2657,5.5224 \times 10^{-2}, 0.6790\right)$ & $-3.1317 \times 10^{-4}$ \\
\hline \multirow[t]{2}{*}{$(0.30,0.05,0.65)$} & $(0.3000,0.0500,0.6500)$ & 0.0000 \\
\hline & $\left(0.3048,2.4285 \times 10^{-2}, 0.6709\right)$ & $-4.9534 \times 10^{-3}$ \\
\hline \multirow[t]{2}{*}{$(0.05,0.10,0.85)$} & $\left(2.1910 \times 10^{-2}, 3.3676 \times 10^{-3}, 0.9747\right)$ & $-4.7349 \times 10^{-2}$ \\
\hline & $(0.0500,0.1000,0.8500)$ & 0.0000 \\
\hline$(0.15,0.10,0.75)$ & $\left(8.3523 \times 10^{-2}, 7.0909 \times 10^{-3}, 0.9094\right)$ & $-2.1739 \times 10^{-2}$ \\
\hline
\end{tabular}




\begin{tabular}{|c|c|c|}
\hline Feed $\left(z_{1}, z_{2}, z_{3}\right)$ & Stationary points $\left(x_{1}, x_{2}, x_{3}\right)$ & $D$ \\
\hline & $(0.1500,0.1000,0.7500)$ & 0.0000 \\
\hline & $(0.1544,0.2908,0.5548)$ & $-1.7323 \times 10^{-2}$ \\
\hline \multirow[t]{3}{*}{$(0.20,0.10,0.70)$} & $\left(0.1257,1.1877 \times 10^{-2}, 0.8624\right)$ & $-1.0321 \times 10^{-2}$ \\
\hline & $(0.2000,0.1000,0.7000)$ & 0.0000 \\
\hline & $(0.2177,0.1838,0.5985)$ & $-1.8376 \times 10^{-3}$ \\
\hline \multirow[t]{3}{*}{$(0.05,0.15,0.80)$} & $\left(1.8972 \times 10^{-2}, 2.1628 \times 10^{-3}, 0.9789\right)$ & $-9.0052 \times 10^{-2}$ \\
\hline & $\left(3.6280 \times 10^{-2}, 0.3989,0.5649\right)$ & $-3.6271 \times 10^{-2}$ \\
\hline & $(0.0500,0.1500,0.8000)$ & 0.0000 \\
\hline \multirow[t]{3}{*}{$(0.10,0.15,0.75)$} & $\left(4.3189 \times 10^{-2}, 3.2072 \times 10^{-3}, 0.9536\right)$ & $-6.5345 \times 10^{-2}$ \\
\hline & $\left(8.9494 \times 10^{-2}, 0.3230,0.5875\right)$ & $-1.1929 \times 10^{-2}$ \\
\hline & $(0.1000,0.1500,0.7500)$ & 0.0000 \\
\hline \multirow[t]{3}{*}{$(0.15,0.15,0.70)$} & $\left(7.3257 \times 10^{-2}, 5.1272 \times 10^{-3}, 0.9216\right)$ & $-4.1534 \times 10^{-2}$ \\
\hline & $(0.1500,0.1500,0.7000)$ & 0.0000 \\
\hline & $(0.1506,0.2353,0.6141)$ & $-1.5662 \times 10^{-3}$ \\
\hline \multirow[t]{3}{*}{$(0.05,0.20,0.75)$} & $\left(1.8393 \times 10^{-2}, 1.7716 \times 10^{-3}, 0.9798\right)$ & -0.1192 \\
\hline & $\left(4.2907 \times 10^{-2}, 0.3218,0.6353\right)$ & $-4.3405 \times 10^{-3}$ \\
\hline & $(0.0500,0.2000,0.7500)$ & 0.0000 \\
\hline \multirow[t]{3}{*}{$(0.10,0.20,0.70)$} & $\left(4.1828 \times 10^{-2}, 2.8004 \times 10^{-3}, 0.9554\right)$ & $-8.2501 \times 10^{-2}$ \\
\hline & $\left(9.6537 \times 10^{-2}, 0.2567,0.6467\right)$ & $-4.2773 \times 10^{-4}$ \\
\hline & $(0.1000,0.2000,0.7000)$ & 0.0000 \\
\hline
\end{tabular}




\begin{tabular}{|c|c|c|}
\hline Feed $\left(z_{1}, z_{2}, z_{3}\right)$ & Stationary points $\left(x_{1}, x_{2}, x_{3}\right)$ & $D$ \\
\hline \multirow[t]{3}{*}{$(0.20,0.20,0.60)$} & $\left(0.1095,9.3104 \times 10^{-3}, 0.8812\right)$ & $-1.4729 \times 10^{-2}$ \\
\hline & $(0.1878,0.1126,0.6996)$ & $1.9549 \times 10^{-3}$ \\
\hline & $(0.2000,0.2000,0.6000)$ & 0.0000 \\
\hline \multirow[t]{2}{*}{$(0.10,0.30,0.60)$} & $\left(4.7113 \times 10^{-2}, 3.3396 \times 10^{-3}, 0.9495\right)$ & $-5.7713 \times 10^{-2}$ \\
\hline & $(0.1080,0.1568,0.7352)$ & $6.8336 \times 10^{-3}$ \\
\hline \multirow[t]{3}{*}{$(0.10,0.50,0.40)$} & $(0.1000,0.3000,0.6000)$ & 0.0000 \\
\hline & $(0.1000,0.5000,0.4000)$ & 0.0000 \\
\hline & $\left(0.1006,1.6405 \times 10^{-2}, 0.8830\right)$ & 0.2374 \\
\hline \multirow[t]{2}{*}{$(0.10,0.55,0.35)$} & $(0.1000,0.5500,0.3500)$ & 0.0000 \\
\hline & $\left(0.1085,3.7935 \times 10^{-3}, 0.8877\right)$ & 0.3639 \\
\hline \multirow[t]{2}{*}{$(0.05,0.60,0.35)$} & $(0.0500,0.6000,0.3500)$ & 0.0000 \\
\hline & $\left(7.0593 \times 10^{-2}, 1.6098 \times 10^{-2}, 0.9133\right)$ & 0.3981 \\
\hline
\end{tabular}

\section{Conclusions}

Using the interval Newton method with the excess Gibbs energy model of NRTL, the phase stability has been predicted for the 10 binary and 2 ternary systems. Although some of the systems are highly non ideal and the local composition model of NRTL may have multiple roots, the interval method has predicted successfully all the stationary points in the tangent plane distance function. 


\section{List of symbols}

D

tangent plane distance

$f(x)$

real nonlinear equation

$g^{\mathrm{E}}$

reduced molar excess Gibbs energy

$g_{\mathrm{m}}$

molar Gibbs energy of mixing surface

$G^{\mathrm{E}}$

molar excess Gibbs energy

$G_{i k}$

NRTL binary interaction parameters

$\Delta G_{\text {mix }}$

Gibbs energy of mixing

$n$

number of components

$R$

gas constant

$T$

absolute temperature

$x$

mole fraction 
feed mole fraction

\section{Greek letters}

$\alpha_{i k}, \alpha_{k i}$

NRTL binary interaction parameters

$\tau_{i k}$

NRTL binary interaction parameters

\section{Subscripts}

$i$

number of components

$k$

number of components

$\mathrm{m}$

mixture

\section{Superscripts}

E

excess

$k$

iteration number 


\section{References}

[1] Y. Demirel and C. McDermott, Chim. Acta Turc. 12 (1984), pp. 173-188.

[2] Y. Demirel and C. McDermott, Chim. Acta Turc. 15 (1987), pp. 329-350.

[3] C. McDermott and Y. Demirel, Rev. Inorg. Chem. 10 (1989), pp. 226-233.

[4] M.L. Michelsen, Fluid Phase Equilibr. 9 (1982), pp. 1-19. Abstract

[5] M.L. Michelsen, Fluid Phase Equilibr. 9 (1982), pp. 21-40. Abstract

[6] C.M. McDonald and C.A. Floudas, AIChE J. 41 (1995), pp. 1798-1817.

[7] A.C. Sun and W.D. Seider, Fluid Phase Equilibr. 103 (1995), pp. 213-249. Abstract

[8] G.A. Iglesias-Silva, A. Bonilla-Petriciolet, P.T. Eubank, J.C. Holste and K.R. Hall, Fluid Phase Equilibr. 210 (2003), pp. 229-245. Abstract

[9] J.Z. Hua, J.F. Brennecke and M.A. Stadtherr, Fluid Phase Equilibr. 116 (1996), pp. 52-59. Abstract

[10] J.Z. Hua, J.F. Brennecke and M.A. Stadtherr, Ind. Eng. Chem. Res. 37 (1998), pp. 1519-1527. Abstract-Compendex $\mid$ Full Text via CrossRef

[11] J.Z. Hua, R.W. Maier, S.R. Tessier, J.F. Brennecke and M.A. Stadtherr, Fluid Phase Equilibr. 158-160 (1999), pp. 607-615. $\underline{\text { Abstract }}$

[12] C.A. Schnepper and M.A. Stadtherr, Comput. Chem. Eng. 20 (1996), pp. 187-199. $\underline{\text { Abstract }}$

[13] S.R. Tessier, J.F. Brennecke and M.A. Stadtherr, Chem. Eng. Sci. 55 (2000), pp. 1785-1796. $\underline{\text { Abstract }}$

[14] C.Y. Gau and M.A. Stadtherr, Comput. Chem. Eng. 26 (2002), pp. 827-840. $\underline{\text { Abstract }}$

[15] M.A. Stadtherr and C.A. Schnepper, AIChE Symp. Ser. 91 (1995), pp. 356-359.

[16] Y. Demirel and H. Gecegörmez, Fluid Phase Equilibr. 65 (1991), pp. 111-133. $\underline{\text { Abstract }}$

[17] L. Lintomen, R.T.P. Pinto, E. Batista, A.J.A. Meirelles and M.R.V. Maciel, J. Chem. Eng. Data 46 (2001), pp. 546-550. Abstract-Compendex | $\underline{\text { Full Text }}$ 